\title{
Electric Vehicle Battery Model Identification and State of Charge Estimation in Real World Driving Cycles
}

\author{
Abbas Fotouhi, Karsten Propp and Daniel J. Auger \\ School of Aerospace, Transport and Manufacturing, Cranfield University \\ College Road, Cranfield, Beds., MK43 0AL, UK \\ Email: a.fotouhi@cranfield.ac.uk
}

\begin{abstract}
This paper describes a study demonstrating a new method of state-of-charge (SoC) estimation for batteries in realworld electric vehicle applications. This method combines realtime model identification with an adaptive neuro-fuzzy inference system (ANFIS). In the study, investigations were carried down on a small-scale battery pack. An equivalent circuit network model of the pack was developed and validated using pulsedischarge experiments. The pack was then subjected to demands representing realistic WLTP and UDDS driving cycles obtained from a model of a representative electric vehicle, scaled match the size of the battery pack. A fast system identification technique was then used to estimate battery parameter values. One of these, open circuit voltage, was selected as suitable for SoC estimation, and this was used as the input to an ANFIS system which estimated the SoC. The results were verified by comparison to a theoretical Coulomb-counting method, and the new method was judged to be effective. The case study used a small 7.2 $\mathrm{V}$ NiMH battery pack, but the method described is applicable to packs of any size or chemistry.
\end{abstract}

Keywords-NiMH Battery; model identification; state of charge estimation; electric vehicle; driving cycle; ANFIS; simulation.

\section{INTRODUCTION}

The development of energy storage systems is key to the process of vehicle electrification. A vital part of battery technology development is the development of insightful modelling and real-time techniques for estimating battery state. From the designer's point of view, good models enable range prediction, for example, which would be impossible without a good model. Perhaps more fundamentally, battery models are vital components of the embedded systems that allow safe operation when charging or discharging, and key enablers in 'pushing the envelope', allowing optimal usage, fast charging and other advanced features.

There are many studies in the literature describing a variety of modelling methods such as electrochemical battery models, mathematical and stochastic models, and electrical circuit models. Among them, equivalent circuit network (ECN) modelling is one of the most common battery modelling approaches as such models are especially suitable for real-time application: having manageable complexity - much less than a full-blown electrochemical model - ECN models have been used in a wide range of applications for many types of battery [1][2]. Such battery models are usually validated through pulse charge/discharge experiments and the evaluation criterion is the model's accuracy to predict battery's terminal voltage from a given current 'input'. However for EV application, SoC estimation is more important than terminal voltage prediction and simple pulse charge/discharge experiments would not be enough to cover all a battery's working conditions. In automotive applications, battery SoC estimation in real world driving conditions is a critical issue and the focus of this study.

In this paper, a procedure is presented for battery testing and implementation in an EV. The procedure consists of the following stages:

1. Battery testing and model extraction,

2. EV modelling and battery model implementation,

3. EV simulation with real-world driving cycles, and

4. SoC estimation under real world working conditions.

Experimental battery tests were conducted using a custombuilt programmable battery discharger. The test setup is suitable for scaled-down battery tests such as single cell or small battery pack tests. The test bench components are the widely-available MATLAB programming environment, an Arduino Uno low-cost processor board, and a simple current sink constructed from readily-available electronics components. The equipment was demonstrated in use on a $7.2 \mathrm{~V} \mathrm{NiMH}$ battery pack containing six cells. Battery model identification was then performed to parameterize an ECN model for the pack using the most widely-used Thevenin model [3], suitable because of its simplicity and speed in realtime applications.

The battery model was then implemented in an EV model for battery duty cycle simulation. The battery performance was investigated for two real world driving cycles: the Worldwide harmonized Light vehicles Test Procedure (WLTP) and the Urban Dynamometer Driving Schedule (UDDS). These simulations gave battery current and terminal voltage, and the battery model was identified during the driving cycle simulations. In this way, the applicability of our identification technique was investigated in a real-time EV application. Finally, on the basis of the identification results, the battery open circuit voltage (OCV) was selected for SoC estimation. An adaptive neuro-fuzzy inference system (ANFIS) was designed for SoC estimation and it was tested for real world driving cycles. The results were also verified using the coulomb-counting method. 


\section{BATTERY MODELLING}

The battery modelling approach consists of the two following main parts:

- Experimental tests on battery pack.

- Extracting battery model's parameters using a system identification technique.

\section{A. Experimental Battery Tests}

As a case study, a six-cell pack of NiMH batteries was tested using the proposed test bench. The battery pack was selected due to its simple and save handling as well as its convenient output voltage. Specifications of the battery pack are listed in Table I. The experiment was conducted by applying consecutive discharge current pulses to the battery and measuring the terminal voltage as the output. The whole test bench is shown in Fig. 1.

TABLE I: NiMH battery pack specifications

\begin{tabular}{|c|c|}
\hline Parameter & Value \\
\hline Rated capacity per cell & $2400 \mathrm{mAh}$ \\
\hline No. of cells & 6 \\
\hline Rated voltage & $7.2 \mathrm{~V}$ \\
\hline Full-Charged voltage & $8.5 \mathrm{~V}$ \\
\hline Cut-off voltage & $6 \mathrm{~V}$ \\
\hline
\end{tabular}

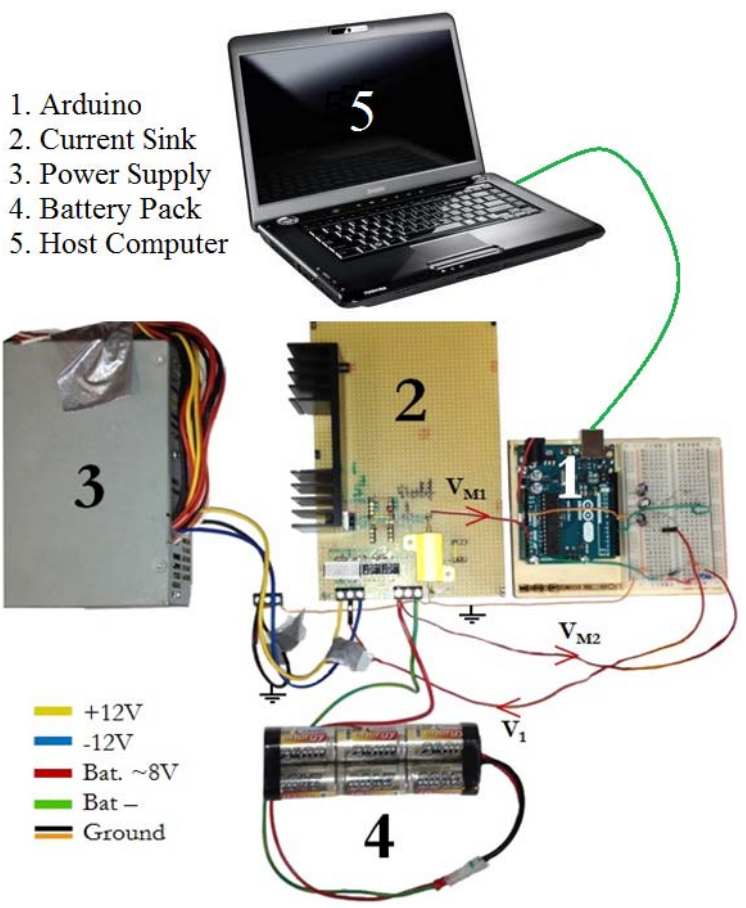

Fig. 1: Battery discharge test bench and NiMH battery pack

The measurement vector includes voltage, current and time values so that other parameters of interest, such as amperehours, can be calculated out of the data. Data is saved in time domain with a sampling rate of one second. Fig. 2 illustrates the battery measurements during an experiment. The test started from fully charged state $(8.5 \mathrm{~V})$ and continued until the terminal voltage dropped below the cut-off voltage $(6 \mathrm{~V})$ which means depleted charge state. Consecutive discharge current pulses are applied to the cell and its terminal voltage is recorded as the output. The discharge rate is $1 \mathrm{C}$ that is $2.4 \mathrm{~A}$ and length of each pulse is 40 seconds with a relaxation time of 60 second in between.
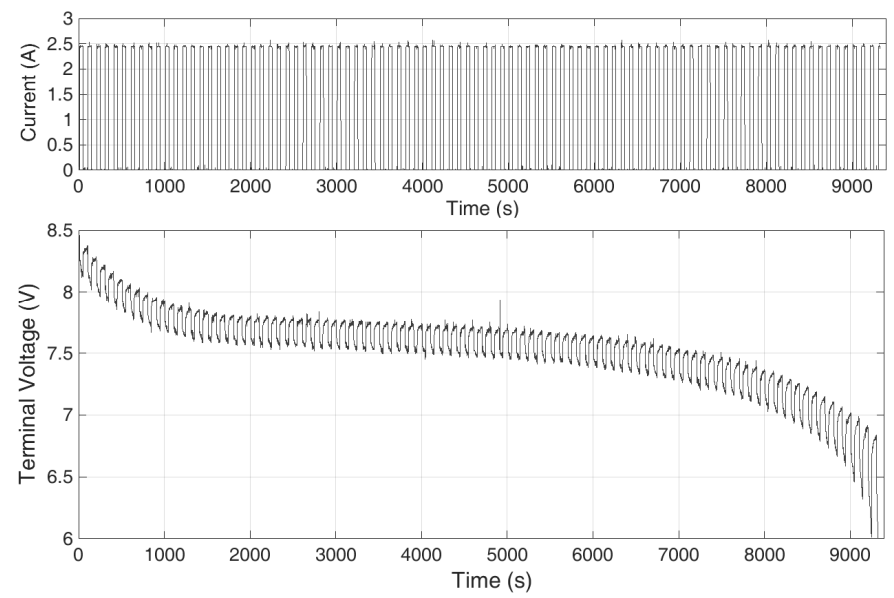

Fig. 2: battery measurements during discharge test

\section{B. Battery Model Identification}

A widely used electrical circuit model called Thevenin model [3] is used as the battery model. The reason of choosing this model is its simplicity and speed which make it applicable in real-time applications. Schematic of Thevenin battery model is illustrated in Fig. 3 in which $V_{t}$ is cell's terminal voltage, $V_{O C}$ is Open Circuit Voltage (OCV), $I_{L}$ is the battery load current, $R_{O}$ is internal ohmic resistance, $R_{P}$ and $C_{P}$ are equivalent polarization resistance and capacitance respectively. Electrical equation of Thevenin model in the frequency domain is as follows:

$$
V_{t}(s)=V_{O C}(s)-I_{L}(s)\left(R_{O}+\frac{R_{P}}{1+R_{P} C_{P} s}\right)
$$

The Prediction-Error Minimization (PEM) algorithm [4] is utilized as the identification algorithm. PEM algorithm is fast enough to be used in real-time battery management system (BMS) for battery model identification. In the identification procedure, the model parameter vector $\theta$ is determined so that the prediction error $\varepsilon$ is minimized. The error is defined as follows:

$$
\varepsilon\left(t_{k}, \theta\right)=y\left(t_{k}\right)-\hat{y}\left(t_{k} \mid t_{k-1} ; \theta\right)
$$

Where $y\left(t_{k}\right)$ is the real output at time $k$ and $\hat{y}\left(t_{k} \mid t_{k-1} ; \theta\right)$ is predicted value of the output at time k using the parameters $\theta$. The prediction error depends on the parameter vector, so an iterative minimization procedure has to be applied. Consequently a scalar fitness function is minimized as follows: 


$$
E_{N}(\theta)=\operatorname{det}\left(\frac{1}{N} \sum_{k=1}^{N} \varepsilon\left(t_{k}, \theta\right) \varepsilon^{T}\left(t_{k}, \theta\right)\right)
$$

In this study, the parameters vector contains all four cell's model parameters. The parameters are optimized so that the least difference between measured terminal voltage $V_{t}$ and model's output is achieved.

$$
\begin{gathered}
\theta=\left[R_{O}, V_{O C}, R_{1}, C_{1}\right] \\
\varepsilon\left(t_{k}, \theta\right)=V_{t}\left(t_{k}\right)-\hat{V}_{t}\left(t_{k} \mid t_{k-1} ; \theta\right)
\end{gathered}
$$

The battery's parameters vary with the state-of-charge (which is constantly changing as current is drawn), so the model's parameters need to be updated in regular time intervals or SoC intervals. Consequently, a short history of battery charge/discharge inside the identification window is used in the calculations. Because of the application of this study in EV energy storage system, SoC identification interval is preferred. Here an identification window of $1 \%$ SoC is used which means that the battery model's parameters are obtained every one percentage change in charge level.

The identified parameters of the battery model are illustrated in Fig. 4. A polynomial is fitted to each of the parameters in order to extract an applicable model. Presenting a polynomial of order $n-1$ with the following formula, the polynomial coefficients $\left(C_{i}\right)$ are stated in TABLE II.

$$
P_{j}=\sum_{i=1}^{n} C_{i} \times(S o C)^{n-i}, \quad j=1,2,3,4
$$

where $P_{j}$ is a parameter of the model and SoC is the charge value in percentage. The polynomial orders are selected based on the identification results which are 7 for OCV and 4 for the other three parameters.

There is a general agreement of the results of this study with previous studies on NiMH battery parameterisation [5]. In addition, the identified model is validated against the experimental data. Fig. 5 demonstrates battery terminal voltage prediction error using the proposed battery model. The average prediction error is $25.5 \mathrm{mV}$ which is roughly $1.3 \%$ of $\mathrm{OCV}$ range.

It should be noted that, battery parameters also depend on other variables such as temperature, battery age, etc. For the sake of simplicity, here the proposed approach is applied to a model which changes just based on SoC. The method can be extended by considering other variables. For example, the temperature effect can be added by using many polynomials obtained at different temperatures. This needs performing similar tests in a range of temperature. For the ageing effect, the identification process should be repeated many times during the battery cycling tests. Consequently, an ageing pattern will be discovered which demonstrates how the polynomials change with regard to the battery age.
TABLE II: NiMH battery model polynomial coefficients

\begin{tabular}{|c|c|c|c|c|}
\hline & $V_{O C}$ & $R_{O}$ & $R_{P}$ & $C_{P}$ \\
\hline $\mathrm{C} 1$ & $4.5664 \mathrm{E}-13$ & $9.486 \mathrm{E}-09$ & $1.466 \mathrm{E}-08$ & $-6.954 \mathrm{E}-05$ \\
\hline $\mathrm{C} 2$ & $-1.4617 \mathrm{E}-10$ & $-2.146 \mathrm{E}-06$ & $-3.016 \mathrm{E}-06$ & $1.357 \mathrm{E}-02$ \\
\hline $\mathrm{C} 3$ & $2.0500 \mathrm{E}-08$ & $1.751 \mathrm{E}-04$ & $2.170 \mathrm{E}-04$ & -0.971 \\
\hline C4 & $-1.6446 \mathrm{E}-06$ & $-6.103 \mathrm{E}-03$ & $-6.504 \mathrm{E}-03$ & 33.501 \\
\hline C5 & $8.2627 \mathrm{E}-05$ & $1.407 \mathrm{E}-01$ & $9.101 \mathrm{E}-02$ & 92.375 \\
\hline C6 & $-2.7297 \mathrm{E}-03$ & - & - & - \\
\hline $\mathrm{C} 7$ & $6.3203 \mathrm{E}-02$ & - & - & - \\
\hline C8 & 6.8305 & - & - & - \\
\hline
\end{tabular}
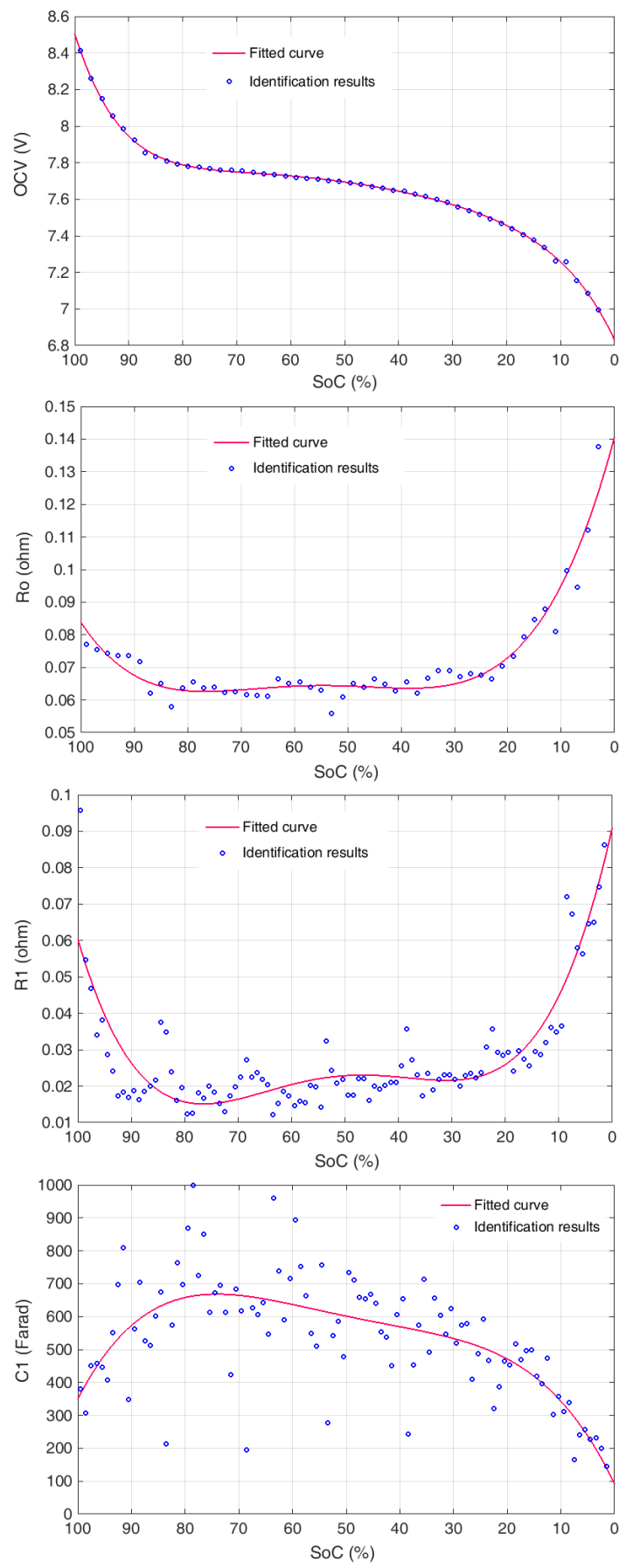

Fig. 4: Identified battery model parameters and fitted polynomials 


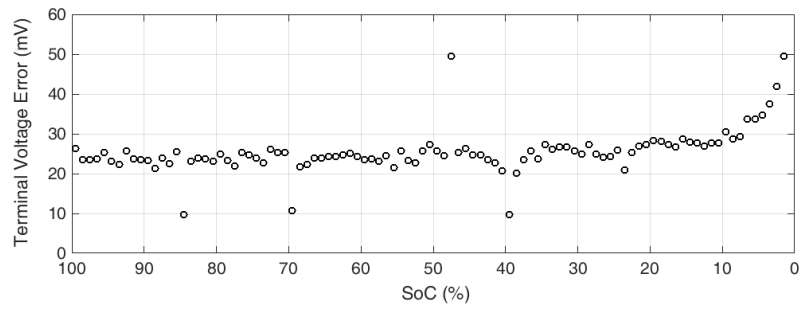

Fig. 5: Battery terminal voltage prediction error using the proposed model

\section{ELECTRIC VeHICLE MOdELLING}

A simulation model has been developed in MATLAB/Simulink environment. The model consists of different parts which are driving cycle, driver model, vehicle dynamics and powertrain. The driving cycle is used as the reference of the vehicle's speed, and a driver model is used to follow it. The driver model is a proportional-integral (PI) controller that tries to follow the reference driving cycle [6].

The vehicle dynamics model includes the calculation of tractive force vs. opposite forces. The tractive force $\left(F_{t}\right)$ is supplied by the electric motor regarding the driver's acceleration command $\left(D_{a}\right)$ as follows:

$$
\begin{aligned}
& F_{t}=\eta_{t} \cdot \frac{G \cdot T_{\mathrm{m}}}{R_{w}} \\
& T_{m}=D_{a} \cdot T_{m, \max }
\end{aligned}
$$

where $\eta_{t}$ is the total efficiency of the powertrain components, $R_{w}$ is the wheel radius, $G$ is the gear ratio, $T_{m}$ is the electric motor's torque and $T_{m \text {,max }}$ is the electric motor's maximum torque at each speed. The tractive force has to overcome the opposite forces including aerodynamic drag force, rolling resistance force, grade force and brake force. The EV model's details are outside the scope of this study and the interested reader will find them in [6]. It should be noted that Nissan Leaf electric vehicle's parameters are used in the simulations based on previous studies in the literature [7],[8].

\section{BATTERY MODEL IDENTIFICATION IN REAL WORLD DRIVING CYCLES}

\section{A. Battery Duty Cycle Simulation}

After the implementation of the battery model in the EV model, battery duty cycle simulation is done on real world driving cycles. The goal of this part is to find out how the algorithms perform under real world driving condition. Battery model identification under various $\mathrm{C}$ rates and frequencies would be different from a simple discharge pulse test.

Two drive cycles are used in the simulations which are WLTP Class 3 and UDDS. The Worldwide harmonized Light vehicles Test Procedure (WLTP), being developed by experts from the European Union, Japan, and India, is a standard for determining the levels of pollutant emissions, fuel or energy consumption, and electric range of light-duty vehicles [9]. WLTP Class 3 is used here which is developed for vehicles with power-weight ratio (kW/Tonne) bigger than 34. Urban Dynamometer Driving Schedule (UDDS) is another driving cycle that is used by U.S. Environmental Protection Agency
(EPA) [10]. UDDS is a dynamometer test on fuel economy of light duty vehicles and it is also known as U.S. FTP-72 (Federal Test Procedure). More details about the driving cycles can be found in [11] and [12].

In the simulations, the power demand is calculated using the EV model. The power demand is then scaled down $(500: 1)$ to be applicable to the NiMH battery pack which consists of six cells. The driving cycles are repeated until the battery is completely depleted. The required current is calculated with regard to demanded power and battery OCV and internal resistance. Applying the current to the battery model, terminal voltage is then obtained. A current controlling algorithm is also designed to prevent the voltage to drop below the cut-off voltage $(6 \mathrm{~V})$ by limiting the current especially at low SoC. The drive cycles, current signal and battery terminal voltage are demonstrated in Fig. 6 and Fig. 7 for WLTP Class 3 and UDDS respectively. In both figures, only one cycle is illustrated whereas the cycles are repeated until the battery is depleted.
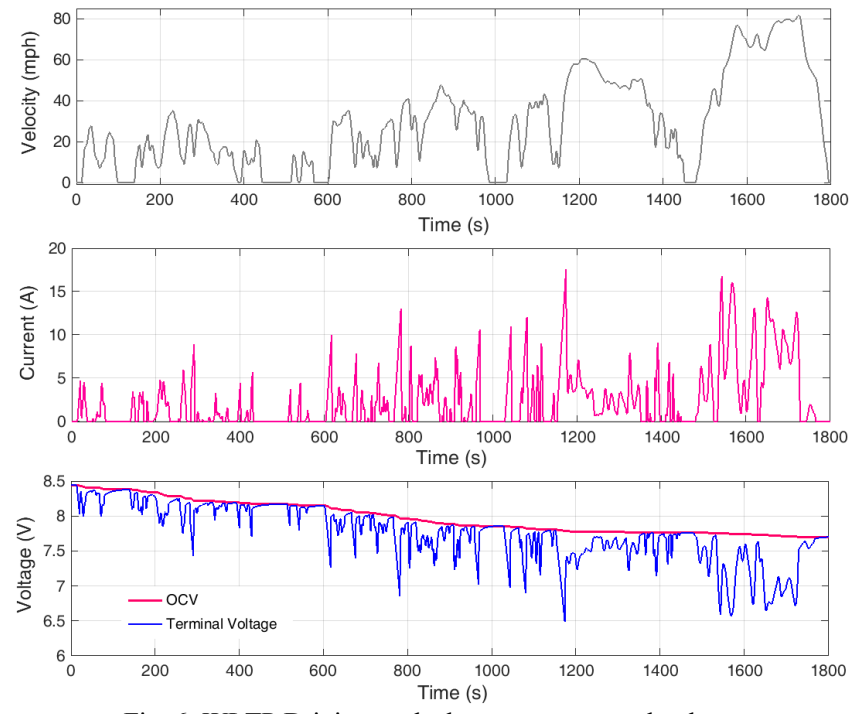

Fig. 6: WLTP Driving cycle, battery current and voltage
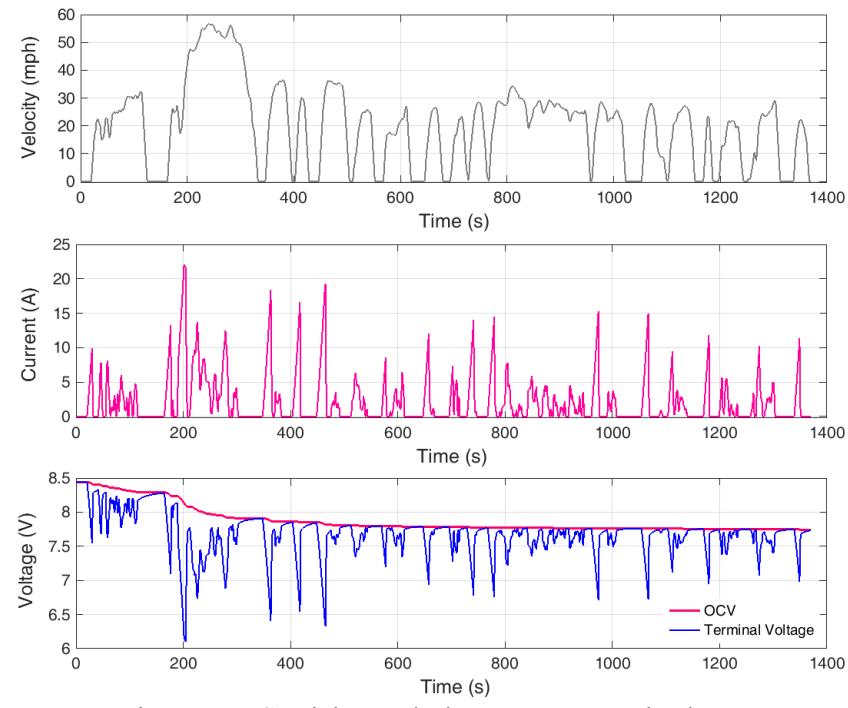

Fig. 7: UDDS Driving cycle, battery current and voltage 


\section{B. Battery identification in real world driving cycles}

Having current and terminal voltage, the battery model can be identified during the driving cycle simulations. At this stage, the goal of identification is different from what was done in section III. This part is focused on investigation of applicability of the identification algorithms in real-time applications for an $\mathrm{EV}$. Since the final target is SoC estimation using battery identification technique, it is not necessary to identify all the model's parameters. In fact, only useful parameters for SoC estimation shall be used, otherwise we are just increasing computational effort in real-time with no gain. After investigating the four parameters of the model based on the identification results, OCV $\left(V_{O C}\right)$ parameter is selected for SoC estimation. The reason is that RC network parameters $\left(R_{1}\right.$ and $C_{1}$ ) have lots of fluctuations (Fig. 4) that is not suitable for SoC estimation. Furthermore, there is a flat region in the ohmic resistance $\left(R_{O}\right)$ curve (Fig. 4) which makes it less suitable for SoC estimation. Consequently, only $\mathrm{OCV}$ is used for $\mathrm{SoC}$ estimation in next part. The identified values of OCV are illustrated in Fig. 8 and Fig. 9 during the simulations on WLTP and UDDS respectively. The results demonstrate that the proposed method is able to track the battery OCV very well even in real world driving cycles. This proves that the proposed method is applicable in real-time applications such as in EV battery management system (BMS).

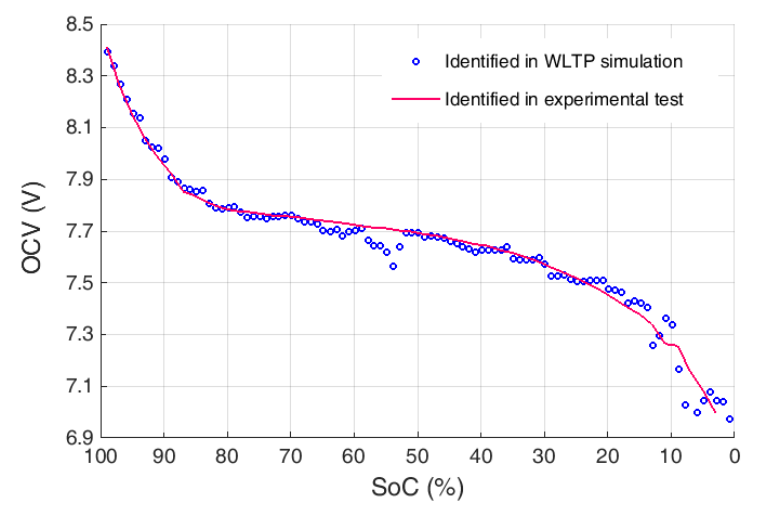

Fig. 8: OCV identification during WLTP simulation and experiment

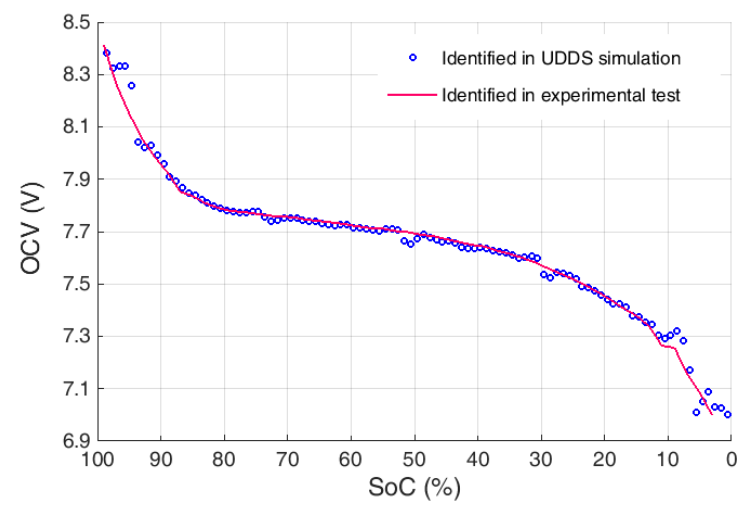

Fig. 9: OCV identification during UDDS simulation and experiment

\section{BATTERy State OF CHARge Estimation}

An Adaptive Neuro-Fuzzy Inference System (ANFIS) model is utilized for SoC estimation. The idea is to find the relationship between the identification results and SoC by using a modelling tool which is ANFIS here. So, the goal of using ANFIS is to find the function $\mathrm{f}$ in the following formula:

$$
S o C=f\left(V_{O C}\right)
$$

So, OCV is obtained in real-time by using the system identification method and ANFIS is utilized for SoC estimation. Schematic of the whole procedure is depicted in Fig. 10 which includes real-time measurement, identification and SoC estimation.

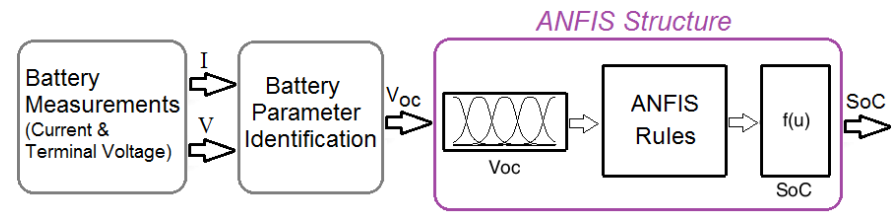

Fig. 10: Battery measurement, identification and SoC estimation

Coulomb-Counting (CC) method is also used here as a benchmark for SoC estimation. It should be noted that $\mathrm{CC}$ method cannot be utilized in practice because it needs proper initial SoC value. In many applications, batteries do not begin to discharge from fully charged state due to self-discharging or being not originally fully charged [13]. So, CC method suffers from accumulated errors caused by wrong initial $\mathrm{SoC}$ value or noise and measurement errors [14],[15]. Another problem is that the battery capacity $\left(C_{t}\right)$ might change under various conditions such as temperature variation which leads to an error in CC method. However, CC method can be used as an ideal reference to evaluate other SoC estimation techniques.

In CC method, SoC is calculated by integrating the load current to know how much capacity is used and remained. Supposing $S_{o} C_{0}$ as the initial SoC at time $t_{0}$, cell's SoC at time $t$ is defined as follows:

$$
S o C=S o C_{0}-\left(\int_{t_{0}}^{t} \frac{\eta i(\tau)}{C_{t}} d \tau\right), \quad 0<S o C<1
$$

where $i(t)$ is the current in ampere (A) and is assumed positive for discharging and negative for charging. Parameter $\eta$ is the battery Coulombic efficiency and $C_{t}$ is cell's total capacity in ampere-second (As) when the time is in second. Therefore, the SoC value is a number between 0 and 1 representing depleted and fully-charged states respectively.

The NiMH battery pack's SoC is calculated using both $\mathrm{CC}$ and ANFIS methods in the simulations. CC method is used as a benchmark to evaluate ANFIS method which is going to be used in real-time. The SoC estimation results are demonstrated in Fig. 11 and Fig. 12 for WLTP and UDDS simulations respectively. As stated in TABLE III, average SoC estimation error is around $2 \%$ whereas the maximum error is less than $10 \%$. 


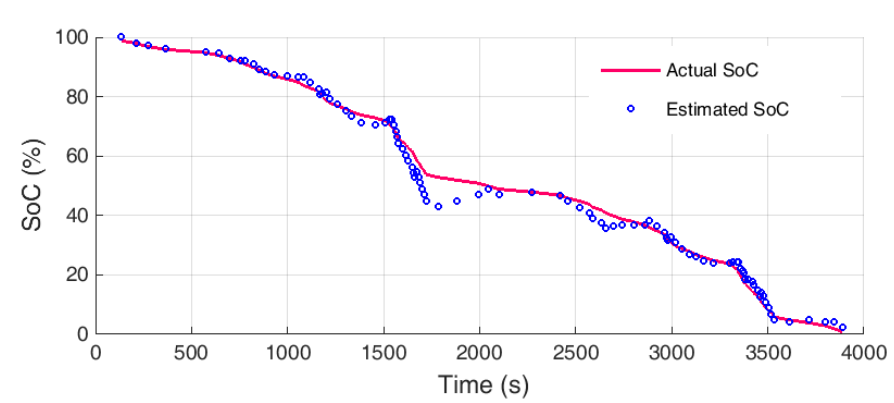

Fig. 11: SoC estimation during WLTP simulation

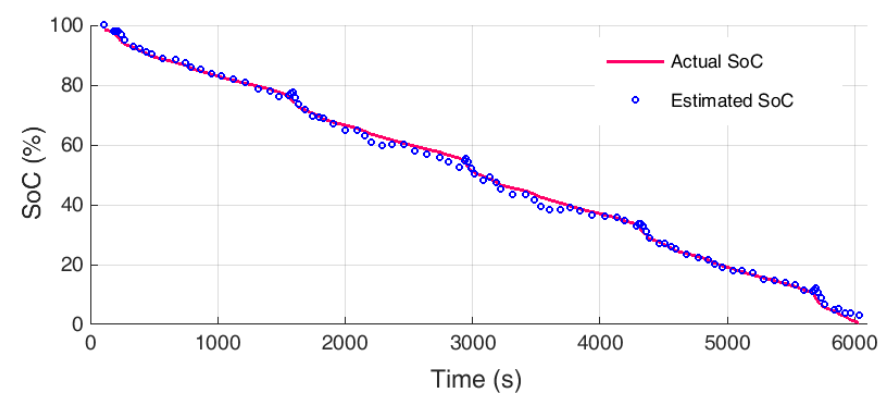

Fig. 12: SoC estimation during UDDS simulation

TABLE III: SoC estimation errors on WLTP and UDDS driving cycles

\begin{tabular}{|c|c|c|}
\hline Driving Cycle & Mean Error & Maximum Error \\
\hline WLTP & $2.13 \%$ & $9.90 \%$ \\
\hline UDDS & $1.12 \%$ & $3.58 \%$ \\
\hline
\end{tabular}

The accuracy of the estimations depends on the identification precision and SoC estimation algorithm both. In other words, an identification error can lead to SoC estimation error too. However, the location of the error occurrence is also important. An identification error in the middle SoC range, between $30 \%$ and $80 \%$, would cause much SoC estimation error since $\mathrm{SoC}$ is more sensitive to $\mathrm{OCV}$ variation in this area.

\section{CONCLUSIONS}

This study has presented a procedure for battery testing and implementation in an EV. In a case study, the procedure was applied to a $7.4 \mathrm{~V}$ NiMH battery pack. The battery model was extracted using a system identification technique and tested with experimental data. The applicability of the proposed technique was then tested under real-world driving conditions. The results demonstrate that the proposed method is able to catch needed information from battery status based on common measurements which are current and terminal voltage. The identification results tell us which battery parameters are the most suitable for SoC estimation and it depends on battery chemistry too. For a NiMH battery, OCV would be enough for SoC estimation. It is also demonstrated that how accuracy of the estimations is affected by the identification precision and SoC estimation algorithm both. Furthermore, it is demonstrated that $\mathrm{SoC}$ is sensitive to the identification results differently in various $\mathrm{SoC}$ regions which is related to battery chemistry. For $\mathrm{NiMH}$ battery, an identification error in the SoC range from $80 \%$ to $30 \%$ would cause much SoC estimation error. Finally, performance of ANFIS is investigated as a battery SoC estimator with average $2 \%$ and maximum $10 \%$ SoC estimation errors.

\section{ACKNOWLEDGMENT}

This research was undertaken as part of the "Revolutionary Electric Vehicle Battery" project, co-funded by Innovate UK. (This project is administered by EPSRC under grant number $\mathrm{EP} / \mathrm{L} 505286 / 1)$. Enquiries for access to the data referred to in this article should be directed to researchdata@cranfield.ac.uk.

\section{REFERENCES}

[1] Marc, T.; Oliver, B.; Dirk, U.S. Development of a voltage-behavior model for NiMH batteries using an impedance-based modeling concept. J. Power Sources 2008, 175, 635-643.

[2] A. Shafiei, A. Momeni, S. Williamson, 2011. Battery Modeling Approaches and Management Techniques for Plug-in Hybrid Electric Vehicles. IEEE Vehicle Power and Propulsion Conference, Chicago, IL.

[3] Z. M. Salameh, M. A. Casacca, W. A. Lynch, 1992. A mathematical model for lead-acid batteries. IEEE Transactions on Energy Conversion 7(1), 93-98.

[4] Ljung, L. J. System Identification: Theory for the User. 2nd ed. New York: Prentice Hall, 1999.

[5] Bornatico, R., A. Storti, L. Mandrioli, A. Zappavigna, Y. Guezennec and G. Rizzoni. "NIMH Battery Characterization and State of Charge Estimation for HEV Applications," Proc. ASME Int. Mechanical Engineering Congress and Exposition, Seattle, 2007. 205-14. doi: 10.1115/IMECE2007-42484.

[6] Fotouhi, A., Auger, D.J., Propp, K., Longo, S. Simulation for prediction of vehicle efficiency, performance, range and lifetime: a review of current techniques and their applicability to current and future testing standards. Hybrid and Electric Vehicles Conference, London, 2014.

[7] G. Choi, T. M. Jahns. "Design of Electric Machines for Electric Vehicles Based on Driving Schedules", in Proceedings of the 2013 IEEE Electric Machines \& Drives Conference. (2013).

[8] J. G. Hayes, R. P. R. de Oliveira, S. Vaughan, M. G. Egan. "Simplified Electric Vehicle Power Train Models and Range Estimation" in Proceedings of the 2011 IEEE Vehicle Power and Propulsion Conference, IEEE, (2011).

[9]https://en.wikipedia.org/wiki/Worldwide_harmonized_Light_vehicles_Test Procedures

[10] http://www.epa.gov/nvfel/testing/dynamometer.htm

[11] A Fotouhi, M Montazeri-Gh, 2013. Tehran driving cycle development using the k-means clustering method. Scientia Iranica 20 (2), 286-293.

[12] A Fotouhi, R Yusof, R Rahmani, S Mekhilef, N Shateri, 2014. A review on the applications of driving data and traffic information for vehicles' energy conservation. Renewable and Sustainable Energy Reviews 37, 822-833.

[13] C.H. Cai, D. Du, Z.Y. Liu, Battery State-of-Charge (SOC) Estimation Using Adaptive Neuro-Fuzzy Inference System (ANFIS), IEEE 2003.

[14] B. Pattipati, C. Sankavaram, and K. Pattipati, "System Identification and Estimation Framework for Pivotal Automotive Battery Management System Characteristics," Systems, Man, and Cybernetics, Part C: Applications and Reviews, IEEE Transactions on, vol. 41, pp. 869-884.

[15] K. Kutluay, Y. Cadirci, Y. S. Ozkazanc, and I. Cadirci. A new online state-of-charge estimation and monitoring system for sealed lead-acid batteries in Telecommunication power supplies. Industrial Electronics, IEEE Transactions on, vol. 52, pp. 1315-1327, 2005. 
2015-09-24

\section{Electric vehicle battery model identification and state of charge estimation in real world driving cycles}

\section{Fotouhi, Abbas}

Institute of Electrical and Electronics Engineers

Abbas Fotouhi, Karsten Propp and Daniel J. Auger. Electric vehicle battery model identification and state of charge estimation in real world driving cycles. Proceedings of the 7th computer science and electronic engineering conference (CEEC 2015), 24-25th September 2015, Colchester, UK.

http://dx.doi.org/10.1109/CEEC.2015.7332732

Downloaded from Cranfield Library Services E-Repository 